\title{
Ameloblastic Carcinoma-Secondary Type (Dedifferentiated), Peripheral
}

National Cancer Institute

\section{Source}

National Cancer Institute. Ameloblastic Carcinoma-Secondary Type (Dedifferentiated), Peripheral. NCI Thesaurus. Code C54299.

A very rare ameloblastic carcinoma that originates from a pre-existing extraosseous benign ameloblastoma. 